\title{
Outlier-Resistant Observer-Based Control for A Class of Networked Systems under Encoding-Decoding Mechanism
}

\author{
Jiahui Li, Zidong Wang, Hongli Dong, and Xiaojian Yi
}

\begin{abstract}
This paper is concerned with a new outlier-resistant observer-based control problem for a class of networked systems (NSs) under the encoding-decoding communication mechanism (EDCM). In order to lighten the communication burden and enhance the data security, the EDCM is introduced in the observerto-controller channel. In case of the measurement outliers, a specific saturation function is adopted in the observer structure to restrain the abnormal innovations so as to mitigate the side effects brought from the outliers. The aim of this paper is to design an observer-based controller such that, in the presence of measurement outliers, the closed-loop NS achieves the input-tostate stability (ISS) under the EDCM. By means of the uniform quantization technique, a criterion is first established in terms of the sizes of the encoding alphabet and the encoding period so as to ensure the detectability of the NS, and the requirement is also given on the capacity of the communication channel at each time instant. Then, with the help of ISS theory, the desired controller is obtained with its gain matrix parameterized by the solution to a certain inequality that can be solved via standard software packages. Finally, the effectiveness of the derived theoretical results is verified through three numerical simulation examples.
\end{abstract}

Index Terms-Networked system, encoding-decoding communication mechanism, outlier-resistant observer, channel capacity, input-to-state stability.

\section{INTRODUCTION}

Over the past several decades, networked systems (NSs) have received considerable research attention due to their prospective applications in various domains including, but are not limited to, unmanned aerial vehicles, remote diagnostics and troubleshooting, space and terrestrial exploration, and factory automation [4], [5], [11], [12], [14], [22], [25], [26], [29]-[31], [34], [36], [44]. In NSs, the information exchanges among actuators, sensors, controllers and estimators/filters are executed over networked media such as wireless or distributed networks [13]. Comparing to the conventional point-to-point communication, the network-based communication enjoys a

This work was supported in part by the National Natural Science Foundation of China under Grants 61933007, 61873148 and 61873058, the Natural Science Foundation of Heilongiiang Province of China under Grant ZD2019F001, the Key Laboratory of Advanced Perception and Intelligent Control of Highend Equipment of Ministry of Education in Anhui Polytechnic University of China under Grant GDSC202016, and the Alexander von Humboldt Foundation of Germany. (Corresponding author: Hongli Dong.)

$\mathrm{J}$. Li and H. Dong are with the Artificial Intelligence Energy Research Institute, Northeast Petroleum University, Daqing 163318, China. They are also with the Heilongjiang Provincial Key Laboratory of Networking and Intelligent Control, Northeast Petroleum University, Daqing 163318, China. (Email: shiningdhl@vip.126.com)

Z. Wang is with the Department of Computer Science, Brunel University London, Uxbridge, Middlesex, UB8 3PH, United Kingdom. (Email: Zidong. Wang@brunel.ac.uk)

$\mathrm{X}$. Yi is with the School of Mechatronical Engineering, Beijing Institute of Technology, Beijing 100081, China. (Email: yixiaojianbit@sina.cn) number of distinctive merits. For example, the introduction of network renders the effectiveness in eliminating unnecessary wiring between system components, which indicates that the complexity and overhead of the corresponding system can be significantly diminished. Moreover, when some system components are required to be added/removed, NSs can be easily modified or upgraded without causing major changes in the system structure.

Despite the popularity of the NSs, the insertion of the communication network does give rise to some inevitable issues such as limited channel capacity and network security which, in turn, result in the so-called network-induced phenomena such as signal quantization [27], packet dropout [16], and communication delays [15]. These phenomena, if not adequately handled, could severely degrade the system performance and this has therefore triggered an ever-increasing research interest in both the analysis and synthesis problems for NSs in the past few decades. For example, many excellent results have been available on the estimation/control problems of NSs subject to network-induced phenomena, see e.g. [8], [32], [37]. From another perspective, to reduce the occurrence of the undesired network-induced phenomena, an active way is to employ certain communication protocols so as to regulate the data transmissions, and some widely deployed protocols include the event-triggering protocol [6], [7], [43], [50], the Round-Robin protocol [28], [35], [49], the try-once-discard protocol [21], [40] and the stochastic communication protocol [45], [48], to name just a few.

It is worth emphasizing that the main idea of the abovementioned communication protocols is to alleviate the channel congestion by reducing the communication frequency and allowing for necessary network traffic only. Serving as yet another effective communication mechanism, the so-called encoding-decoding communication mechanism (EDCM) aims to send the symbolic data (rather than the original data) through communication channels to realize data compression. The employment of the EDCM not only reduces resource occupation but also enhances data security in the process of data transmission. Note that some pioneering EDCM-related work has been available in the literature, see e.g. [23], [24], [41], [42]. For instance, a sufficient condition has been presented in [23] to stabilize the linear time-invariant system by using the sampled encoded states/outputs. The EDCM has been further applied in [41] to research into the synchronization control problem for a kind of dynamical networks subject to packet dropouts, where a decoder-based controller has been constructed to guarantee the synchronization of the dynamical networks. 
In engineering practice, the measurement output may undergo abrupt yet large disturbances, which are referred to as measurement outliers, for a variety of reasons such as operation errors, sensor noises/failures, and unknown environmental disturbances. Up to now, the outlier detection approaches and the state-of-the-art outlier resistant/resilient control schemes have received particular research attention [17]. Different from the measurement noises [20], the measurement outliers may lead to abnormal magnitude changes which, if directly utilized in the observer/estimator implementation, would result in abnormal innovation and subsequently deteriorate the performance of the observer/estimator [46], [47]. As such, it makes practical sense to look into the outlier-resistant state estimation issue by removing/restraining the side-effects caused by measurement outliers, see [1]-[3], [10], [33], [38] for some initial results. In [1], a modified maximum likelihood estimator has been set up which is robust to the possible outliers. Moreover, in [10], a new Kalman filter has been put forward that is insensitive to measurement outliers. Nonetheless, when it comes to the observer-based control problems, the corresponding results have been very few and the main objective of this paper is therefore to propose an outlier-resistant observer to weaken the influence from the measurement outliers while maintaining the desired control performance.

Motivated by the above discussions, in this paper, we are concerned with the outlier-resistant observer-based control problem for a class of nonlinear NSs under EDCM. In doing so, three challenges we have to face are identified as follows: 1) how to establish a unified control-theoretic framework that takes the EDCM and measurement outliers into simultaneous consideration? 2) how to design an effective observer to mitigate the side-effects from the measurement outliers on the estimation performance? and 3) how to handle the decoding error (between the decoding system state and the actual system state) and use the incomplete decoding information to accomplish the desired control task?

To overcome the identified challenges, we make dedicated efforts in this paper with certain distinctive features outlined as follows: 1) a specific saturation function is introduced into the observer structure so as to attenuate the side-effects of the measurement outliers on the observation error; 2) a suitable EDCM is proposed for the addressed NSs to reduce communication resource occupation and also enhance data transmission security; 3) the combined effects from the encoding period as well as the size of encoding alphabets are analyzed in a quantitative way, and the requirement is given on the channel capacity is given; and 4) an easyto-implement controller design algorithm is developed with explicitly characterized controller gain by using the ISS theory.

The rest of this paper is structured as follows. In Section II, the problem to be addressed is presented and the basic idea of the EDCM is introduced. In Section III, the encoding-decoding-based detectability is analyzed and the desired outlier-resistant observer-based controller is designed. In Section IV, three simulation examples are provided and Section $\mathrm{V}$ concludes this paper.

Notation: The notation used here is fairly standard. $I$ and 0 represent the identity matrix and zero matrix with proper dimensions, respectively. $\lambda_{\max }(A)\left(\lambda_{\min }(A)\right)$ is the maximum (minimum) eigenvalue of a symmetric matrix $A$. For a matrix $B, B^{\perp}$ is an orthogonal basis of the null space for matrix $B^{T}$, i.e., $B^{T} B^{\perp}=0 .\|y\|_{2}$ and $\|y\|_{\infty}$ represent the Euclidean norm and infinite norm of vector $y$, respectively. For a given real number $x,\lceil x\rceil$ denotes the least integer greater than or equal to $x$. In symmetric block matrices, " $*$ " is used as an ellipsis for terms induced by symmetry. If $\gamma(\cdot): \mathbb{R}^{+} \mapsto \mathbb{R}^{+}$is a continuous strictly increasing function with $\gamma(0)=0$, then $\gamma(\cdot)$ is called as a $\mathcal{K}$ class function. Further, if $\gamma(\cdot) \in \mathcal{K}$ with $\gamma(s) \rightarrow \infty$ as $s \rightarrow \infty$, we say that $\gamma(\cdot)$ is a $\mathcal{K}_{\infty}$ class function. A function $\Im(\cdot, \cdot): \mathbb{R}^{+} \times \mathbb{R}^{+} \mapsto \mathbb{R}^{+}$is said to be of class $\mathcal{K} \mathcal{L}$, if the mapping $\Im(s, k)$ is of class $\mathcal{K}$ for each fixed $k$, and is decreasing to 0 as $k \rightarrow \infty$ for each fixed $s$.

\section{Problem Formulation And Preliminaries}

Consider an NS shown in Fig. 1, where the data delivery between the observer and the controller is implemented via a network under a certain EDCM. The state estimates are encoded to certain codewords by a designed encoder, and then the received codewords are decoded by the corresponding decoder at the controller side. In the following, the physical plant, the EDCM, the observer and the controller will be presented in the state space.

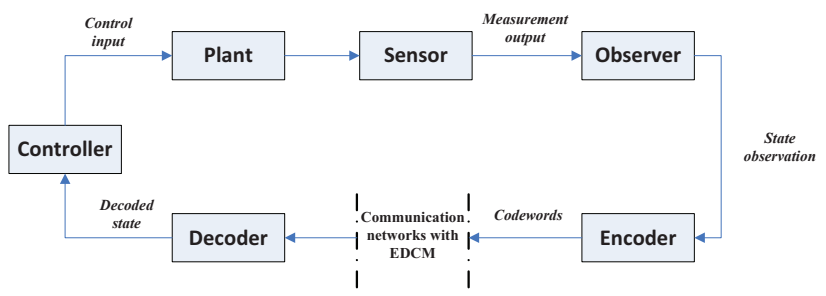

Fig. 1. Structure of the NS under EDCM.

\section{A. The physical plant}

In this paper, we consider the following class of nonlinear discrete-time systems:

$$
\left\{\begin{aligned}
x_{k+1} & =E x_{k}+D f\left(x_{k}\right)+B u_{k} \\
y_{k} & =N x_{k} \\
x_{0} & =s_{0}
\end{aligned}\right.
$$

where $x_{k} \in \mathbb{R}^{n_{x}}$ is the system state with the initial condition $x_{0}=s_{0}$ satisfying $\left\|s_{0}\right\|_{2} \leq \epsilon_{0}$, and $\epsilon_{0}$ is a known constant. $y_{k} \in \mathbb{R}^{n_{y}}$ denotes the measurement output. $u_{k} \in \mathbb{R}^{n_{u}}$ is the control input signal. $f(\cdot): \mathbb{R}^{n_{x}} \mapsto \mathbb{R}^{n_{x}}$ is a nonlinear vector-valued function. $E, D, B$ and $N$ are known real-valued matrices with compatible dimensions.

Assumption 1: The nonlinear vector-valued function $f(\cdot)$ satisfies $f(0)=0$ and

$$
\left\|f\left(x_{k}+\delta_{k}\right)-f\left(x_{k}\right)\right\|_{2} \leq\left\|U \delta_{k}\right\|_{2}
$$

where $U$ is a known real matrix of appropriate dimensions and $\delta_{k} \in \mathbb{R}^{n_{x}}$ is a vector. 


\section{B. The outlier-resistant observer}

The objective of this subsection is to design an observer on the basis of the available measurements. As discussed in the introduction, measurement outliers do exist which, if not properly dealt with, would deteriorate the observer performance or even destabilize the error dynamics. To resolve such a problem, a so-called outlier-resistant observer is constructed as follows:

$$
\left\{\begin{aligned}
\hat{x}_{k+1} & =E \hat{x}_{k}+D f\left(\hat{x}_{k}\right)+B u_{k}+K_{e} \sigma\left(y_{k}-N \hat{x}_{k}\right) \\
\hat{x}_{0} & =0
\end{aligned}\right.
$$

where $\hat{x}_{k} \in \mathbb{R}^{n_{x}}$ is the state estimate and $K_{e}$ is the observer gain to be designed. Here, the purposely introduced saturation function $\sigma(\cdot): \mathbb{R}^{n_{y}} \mapsto \mathbb{R}^{n_{y}}$ is defined as follows:

$$
\sigma(\varpi)=\left[\begin{array}{llll}
\sigma_{1}^{T}\left(\varpi_{1}\right) & \sigma_{2}^{T}\left(\varpi_{2}\right) & \cdots & \sigma_{n_{y}}^{T}\left(\varpi_{n_{y}}\right)
\end{array}\right]^{T}
$$

with $\sigma_{\iota}\left(\varpi_{\iota}\right) \triangleq \operatorname{sign}\left(\varpi_{\iota}\right) \min \left\{\varpi_{\iota, \max },\left|\varpi_{\iota}\right|\right\}, \iota=1,2, \ldots, n_{y}$, where $\varpi_{\iota, \max }$ is the $\iota$ th element of $\varpi_{\max }$ (i.e., the saturation level).

Denoting $e_{k} \triangleq x_{k}-\hat{x}_{k}$ and $\tilde{f}\left(e_{k}\right) \triangleq f\left(x_{k}\right)-f\left(\hat{x}_{k}\right)$, we obtain the corresponding error dynamics as follows:

$$
e_{k+1}=E e_{k}+D \tilde{f}\left(e_{k}\right)-K_{e} \sigma\left(N e_{k}\right) .
$$

Remark 1: As a kind of contaminated measurements, the outliers are meant to deviate significantly from the normal values that have recently stirred quite a lot research attention. Measurement outliers can be caused by a variety of reasons such as sensor aging/failures, operational errors and environmental factors. In the context of state estimation, measurement outliers may lead to the calculation of abnormal innovation which would therefore have an adverse effect on the performance of observer. As such, we endeavor to propose an effective observer design scheme so as to prevent outliers from deteriorating the estimation accuracy.

Remark 2: The measurement outliers, if directly utilized in the innovation $y_{k}-N \hat{x}_{k}$, are likely to lead to abnormal deviation of the innovation from its usual pattern, thereby worsening estimation performance. Therefore, a specific saturation function $\sigma(\cdot)$ is introduced in the observer structure so as to constrain the innovation within a predefined range that can be determined a priori according to engineering practice. The proposed observer is referred to as an outlierresistant one capable of attenuating the negative effects from the measurement outliers. It is worth mentioning that the proposed observer (3) will be degenerated into the traditional Luenberger-like one when the saturation level goes to infinity.

\section{A general encoding-decoding procedure}

In order to reduce the communication resource occupation and realize data transmission security, we are now in a position to introduce the EDCM in the observer-to-controller channel. To proceed further, let us present the general form of the encoding-decoding procedure for system (1).

The general form of the encoder is described by

$$
\theta(l d)=\mathcal{J}\left(\hat{x}_{l d}\right)
$$

and the decoder is described by

$$
\breve{x}_{l d}=\mathcal{F}(\theta(l d))
$$

where $\theta(l d)(l=1,2, \ldots)$ is the codeword generated at the encoding instant $l d$ by the encoder, $d$ is a constant representing the encoding period, $\breve{x}_{l d}$ is the decoded value of $\hat{x}_{l d}$ at time instant $l d$, and $\mathcal{J}(\cdot)$ and $\mathcal{F}(\cdot)$ are, respectively, encoder and decoder functions to be designed later.

\section{Uniform quantization approach}

As a critical part in the development of EDCM, the uniform quantization approach is briefly introduced as follows.

For given scale parameter $c>0$ and integer $q$, we divide the hyperrectangle $\mathcal{B}_{c}=\left\{\zeta \in \mathbb{R}^{n_{x}}:\|\zeta\|_{\infty} \leq c, i=1,2, \ldots, n_{x}\right\}$ into $q^{n_{x}}$ hyperrectangles $\mathbb{I}_{\varepsilon_{1}}^{1}(c) \times \mathbb{I}_{\varepsilon_{2}}^{2}(c) \times \cdots \times \mathbb{I}_{\varepsilon_{n_{x}}}^{n_{x}}(c)$, where $\varepsilon_{1}, \varepsilon_{2}, \ldots, \varepsilon_{n_{x}} \in\{1,2, \ldots, q\}$ and

$$
\begin{aligned}
\mathbb{I}_{1}^{i}(c) \triangleq & \left\{\zeta_{i} \mid-c \leq \zeta_{i}<-c+2 c / q\right\}, \\
\mathbb{I}_{2}^{i}(c) \triangleq & \left\{\zeta_{i} \mid-c+2 c / q \leq \zeta_{i}<-c+4 c / q\right\}, \\
& \vdots \\
\mathbb{I}_{q}^{i}(c) \triangleq & \left\{\zeta_{i} \mid c-2 c / q \leq \zeta_{i}<c\right\} .
\end{aligned}
$$

with $\zeta_{i}$ being the $i$ th element of the vector $\zeta$. The center of the hyperrectangle $\mathbb{I}_{\varepsilon_{1}}^{1}(c) \times \mathbb{I}_{\varepsilon_{2}}^{2}(c) \times \cdots \times \mathbb{I}_{\varepsilon_{n_{x}}}^{n_{x}}(c)$ is defined as

$$
\hbar_{c}\left(\varepsilon_{1}, \varepsilon_{2}, \ldots, \varepsilon_{n_{x}}\right) \triangleq\left[\begin{array}{c}
-c+\frac{\left(2 \varepsilon_{1}-1\right) c}{q} \\
-c+\frac{\left(2 \varepsilon_{2}-1\right) c}{q} \\
\vdots \\
-c+\frac{\left(2 \varepsilon_{n_{x}}-1\right) c}{q}
\end{array}\right] .
$$

Consequently, for any $\zeta \in \mathcal{B}_{c}$, there exist unique integers $\varepsilon_{1}, \varepsilon_{2}, \ldots, \varepsilon_{n_{x}} \in\{1,2, \ldots, q\}$ such that $\zeta \in \mathbb{I}_{\varepsilon_{1}}^{1}(c) \times \mathbb{I}_{\varepsilon_{2}}^{2}(c) \times$ $\cdots \times \mathbb{I}_{\varepsilon_{n_{x}}}^{n_{x}}(c)$, which implies

$$
\left\|\zeta-\hbar_{c}\left(\varepsilon_{1}, \varepsilon_{2}, \ldots, \varepsilon_{n_{x}}\right)\right\|_{2} \leq \frac{\sqrt{n_{x}} c}{q} .
$$

For subsequent analysis, the following definitions are given. Definition 1: [9] For a nonlinear function $\phi(\cdot): \mathbb{R}^{n_{y}} \mapsto$ $\mathbb{R}^{n_{y}}$ and real matrices $W_{1}, W_{2} \in \mathbb{R}^{n_{y} \times n_{y}}$ where $W=W_{2}-$ $W_{1}$ is a positive definite matrix, if

$$
\left(\phi(\varpi)-W_{1} \varpi\right)^{T}\left(\phi(\varpi)-W_{2} \varpi\right) \leq 0, \forall \varpi \in \mathbb{R}^{n_{y}}
$$

is true, then $\phi(\cdot)$ is said to satisfy a sector condition and belongs to the sector $\left[W_{1}, W_{2}\right]$.

Definition 2: [41] The nonlinear discrete-time system (1) is said to be detectable if there exist families of encoder-decoder pairs (5) and (6) with an encoding alphabet $\mathcal{H}$ of size $\mathcal{X}$ such that

$$
\lim _{k \rightarrow \infty}\left\|x_{k}-\breve{x}_{k}\right\|_{2}=0
$$

holds for any solution of (1).

Definition 3: [18] Consider a nonlinear discrete-time system with the following form:

$$
\rho_{k+1}=g\left(\rho_{k}, \nu_{k}\right)
$$

where $\rho_{k} \in \mathbb{R}^{n}, \nu_{k} \in \mathbb{R}^{p}$, and $g(\cdot, \cdot): \mathbb{R}^{n} \times \mathbb{R}^{p} \mapsto \mathbb{R}^{n}$ stand for the system state, exogenous input, and continuous nonlinear function with $g(0,0)=0$, respectively. For system (11), if 
there exist a $\mathcal{K} \mathcal{L}$ function $\alpha(\cdot, \cdot)$ and a $\mathcal{K}$ class function $\beta(\cdot)$ such that the following condition

$$
\left\|\rho_{k}\right\|_{2} \leq \alpha\left(\left\|\rho_{0}\right\|_{2}, k\right)+\beta\left(\left\|\nu_{k}\right\|_{\infty}\right)
$$

holds for $\forall k \geq 0$ and $\forall \rho_{0} \in \mathbb{R}^{n}$ where $\left\|\nu_{k}\right\|_{\infty} \triangleq \sup _{k}\left\{\left\|\nu_{k}\right\|\right\}$, then the system (11) is said to be input-to-state stable.

This paper aims to lay emphasis on the control problem based on an outlier-resistant observer for the system (1) under the EDCM. The objective of this paper is to develop an efficient encoding-decoding procedure (5)-(6) and an outlierresistant observer-based controller such that 1 ) the underlying system (1) is detectable; and 2) the closed-loop system is input-to-state stable.

\section{Main Results}

\section{A. Detectability Analysis}

In this subsection, we are ready to examine the detectability issue, that is, whether and how the encoded data could be recovered from the codewords with a prescribed accuracy constraint. To start with, we give the following lemmas that will be used in deriving our main results in the sequel.

Lemma 1: [9] There exist diagonal matrices $M_{1}$ and $M_{2}$ satisfying $0 \leq M_{1}<I \leq M_{2}$ such that the saturation function $\sigma\left(N e_{k}\right)$ in (4) can be divided into a linear part and a nonlinear part as

$$
\sigma\left(N e_{k}\right)=M_{1} N e_{k}+\phi\left(N e_{k}\right)
$$

where $\phi(\cdot)$ is a nonlinear vector-valued function that satisfies the sector condition with $W_{1}=0$ and $W_{2}=M$, where $M=$ $M_{2}-M_{1}$, i.e., $\phi\left(N e_{k}\right)$ satisfies the following inequality:

$$
\phi^{T}\left(N e_{k}\right)\left[\phi\left(N e_{k}\right)-M N e_{k}\right] \leq 0 .
$$

Lemma 2: Let the scalar $\varrho_{1}>0$ be given. If there exist a positive definite matrix $P>0$ and a scalar $\mu_{1}>0$ such that the following linear matrix inequality (LMI)

$$
\tilde{\Xi}=\left[\begin{array}{ccc}
\tilde{\Xi}_{11} & * & * \\
0 & -\mu_{1} I & * \\
P E & P D & -P
\end{array}\right]<0
$$

holds for any two solutions $x_{k}^{1}$ and $x_{k}^{2}$ of (1), then we have

$$
\left\|x_{k+1}^{1}-x_{k+1}^{2}\right\|_{2}<\gamma_{1}\left\|x_{k}^{1}-x_{k}^{2}\right\|_{2}
$$

where

$$
\begin{aligned}
\tilde{\Xi}_{11} & \triangleq-\left(1+\varrho_{1}\right) P+\mu_{1} U^{T} U, \\
\gamma_{1} & \triangleq \sqrt{\left(\left(1+\varrho_{1}\right) \lambda_{\max }(P)\right) / \lambda_{\min }(P)} .
\end{aligned}
$$

Proof: Denoting $v_{k} \triangleq x_{k}^{1}-x_{k}^{2}$, we easily obtain that $v_{k+1}=E v_{k}+D f\left(x_{k}^{1}\right)-D f\left(x_{k}^{2}\right)$.

Consider the following Lyapunov function:

$$
V_{k}=v_{k}^{T} P v_{k}
$$

and it follows from $\triangle V_{k}=V_{k+1}-V_{k}$ that

$$
\begin{aligned}
\triangle V_{k}-\varrho_{1} V_{k}= & {\left[E v_{k}+D f\left(x_{k}^{1}\right)-D f\left(x_{k}^{2}\right)\right]^{T} P } \\
& \times\left[E v_{k}+D f\left(x_{k}^{1}\right)-D f\left(x_{k}^{2}\right)\right] \\
& -\left(1+\varrho_{1}\right) v_{k}^{T} P v_{k} .
\end{aligned}
$$

In terms of Assumption 1, we have

$$
\begin{aligned}
& \triangle V_{k}-\varrho_{1} V_{k} \\
\leq & v_{k}^{T}\left[E^{T} P E-\left(1+\varrho_{1}\right) P\right] v_{k} \\
& +2 v_{k}^{T} E^{T} P D\left(f\left(x_{k}^{1}\right)-f\left(x_{k}^{2}\right)\right) \\
& +\left(f\left(x_{k}^{1}\right)-f\left(x_{k}^{2}\right)\right)^{T} D^{T} P D\left(f\left(x_{k}^{1}\right)-f\left(x_{k}^{2}\right)\right) \\
& -\mu_{1}\left(f\left(x_{k}^{1}\right)-f\left(x_{k}^{2}\right)\right)^{T}\left(f\left(x_{k}^{1}\right)-f\left(x_{k}^{2}\right)\right) \\
& +\mu_{1} v_{k}^{T} U^{T} U v_{k} \\
= & \eta_{1 k}^{T} \Xi \eta_{1 k}
\end{aligned}
$$

where

$$
\begin{aligned}
\eta_{1 k} & \triangleq\left[\begin{array}{cc}
v_{k}^{T} & f^{T}\left(x_{k}^{1}\right)-f^{T}\left(x_{k}^{2}\right)
\end{array}\right]^{T}, \\
\Xi & \triangleq\left[\begin{array}{cc}
\Xi_{11} & * \\
D^{T} P E & D^{T} P D-\mu_{1} I
\end{array}\right], \\
\Xi_{11} & \triangleq E^{T} P E-\left(1+\varrho_{1}\right) P+\mu_{1} U^{T} U .
\end{aligned}
$$

Applying Schur Complement Lemma to (14), it is not difficult to see that $\Xi<0$, which means $V_{k+1}<\left(1+\varrho_{1}\right) V_{k}$. Therefore, we have

$$
\lambda_{\min }(P)\left\|v_{k+1}\right\|_{2}^{2}<\left(1+\varrho_{1}\right) \lambda_{\max }(P)\left\|v_{k}\right\|_{2}^{2} .
$$

and subsequently

$$
\left\|v_{k+1}\right\|_{2}<\gamma_{1}\left\|v_{k}\right\|_{2}
$$

which ends the proof.

Lemma 3: Let a positive scalar $\varrho_{2}<1$ be given and consider the error system (4). Assume that there exist a positive definite matrix $R>0$, a matrix $X$ and a positive scalar $\mu_{2}>$ satisfying

$$
\tilde{\Lambda}=\left[\begin{array}{cccc}
\tilde{\Lambda}_{11} & * & * & * \\
0 & -\mu_{2} I & * & * \\
\mu_{0} \frac{M N}{2} & 0 & -\mu_{0} I & * \\
R E-X M_{1} N & R D & -X & -R
\end{array}\right]<0
$$

where $\tilde{\Lambda}_{11} \triangleq-\left(1-\varrho_{2}\right) R+\mu_{2} U^{T} U$. Let $d \in \mathbb{Z}^{+}$be any positive integer such that $0<\gamma_{2}<1$ where

$$
\gamma_{2} \triangleq \sqrt{\left(\left(1-\varrho_{2}\right)^{d} \lambda_{\max }(R)\right) / \lambda_{\min }(R)} .
$$

Then, we have

$$
\left\|x_{k+d}-\hat{x}_{k+d}\right\|_{2}<\gamma_{2}\left\|x_{k}-\hat{x}_{k}\right\|_{2} .
$$

In this case, the desired observer gain matrix is calculated by $K_{e}=R^{-1} X$.

Proof: For the error dynamics (4), we construct the following Lyapunov function

$$
V_{k}=e_{k}^{T} R e_{k}
$$

and then obtain

$$
\begin{aligned}
\triangle V_{k}+\varrho_{2} V_{k}= & {\left[E e_{k}+D \tilde{f}\left(e_{k}\right)-K_{e} \sigma\left(N e_{k}\right)\right]^{T} R } \\
& \times\left[E e_{k}+D \tilde{f}\left(e_{k}\right)-K_{e} \sigma\left(N e_{k}\right)\right] \\
& -\left(1-\varrho_{2}\right) e_{k}^{T} R e_{k} .
\end{aligned}
$$

In light of (2) and (13), we further derive that

$$
\triangle V_{k}+\varrho_{2} V_{k}=\left[E e_{k}+D \tilde{f}\left(e_{k}\right)-K_{e} \sigma\left(N e_{k}\right)\right]^{T} R
$$




$$
\begin{aligned}
& \times\left[E e_{k}+D \tilde{f}\left(e_{k}\right)-K_{e} \sigma\left(N e_{k}\right)\right] \\
& -\left(1-\varrho_{2}\right) e_{k}^{T} R e_{k}-\mu_{2} \tilde{f}^{T}\left(e_{k}\right) \tilde{f}\left(e_{k}\right) \\
& +\mu_{2} e_{k}^{T} U^{T} U e_{k}-\mu_{0} \phi^{T}\left(N e_{k}\right) \phi\left(N e_{k}\right) \\
& +\mu_{0} \phi^{T}\left(N e_{k}\right) M N e_{k} \\
= & \eta_{2 k}^{T} \Lambda \eta_{2 k}
\end{aligned}
$$

where

$$
\begin{aligned}
\eta_{2 k} & \triangleq\left[\begin{array}{lll}
e_{k}^{T} & \tilde{f}^{T}\left(e_{k}\right) & \phi^{T}\left(N e_{k}\right)
\end{array}\right]^{T}, \\
\Lambda & \triangleq\left[\begin{array}{ccc}
\Lambda_{11} & * & * \\
\Lambda_{21} & D^{T} R D-\mu_{2} I & * \\
\Lambda_{31} & -K_{e}^{T} R D & K_{e}^{T} R K_{e}-\mu_{0} I
\end{array}\right], \\
\Lambda_{11} & \triangleq\left(E-K_{e} M_{1} N\right)^{T} R\left(E-K_{e} M_{1} N\right)+\tilde{\Lambda}_{11}, \\
\Lambda_{21} & \triangleq D^{T} R\left(E-K_{e} M_{1} N\right), \\
\Lambda_{31} & \triangleq-K_{e}^{T} R\left(E-K_{e} M_{1} N\right)+\mu_{0} \frac{M N}{2} .
\end{aligned}
$$

Applying Schur Complement Lemma to (18) yields $\triangle V_{k}+$ $\varrho_{2} V_{k}<0$, which implies

$$
V_{k+d}<\left(1-\varrho_{2}\right)^{d} V_{k}
$$

where $d$ is any positive integer such that $0<\gamma_{2}<1$. Furthermore, we have

$$
\lambda_{\min }(R)\left\|e_{k+d}\right\|_{2}^{2}<\left(1-\varrho_{2}\right)^{d} \lambda_{\max }(R)\left\|e_{k}\right\|_{2}^{2}
$$

or

$$
\left\|x_{k+d}-\hat{x}_{k+d}\right\|_{2}<\gamma_{2}\left\|x_{k}-\hat{x}_{k}\right\|_{2},
$$

which ends the proof.

In what follows, we shall present an encoding-decoding procedure and analyze the detectability of (1). Drawing on the ideas of EDCM in [42], we know that the decoded state $\breve{x}_{k}$ cannot be directly utilized to construct state observer (3) due to the constraint on the network bandwidth. Therefore, an auxiliary state $\bar{x}_{k}$ is introduced on the observer side which will be defined later. For the time being, the error vector at the encoding instant $l d$ is denoted by $\zeta(l d) \triangleq \hat{x}_{l d}-\bar{x}_{l d}$.

In terms of Lemmas 2 and 3 for certain constants $\gamma_{1}$ and $\gamma_{2}$, the following encoding-decoding procedure is designed for (1).

Encoder: For $\zeta(l d) \triangleq \hat{x}_{l d}-\bar{x}_{l d} \in \mathbb{I}_{\varepsilon_{1}}^{1}(c(l d)) \times \mathbb{I}_{\varepsilon_{2}}^{2}(c(l d)) \times$ $\cdots \times \mathbb{I}_{\varepsilon_{n_{x}}}^{n_{x}}(c(l d)) \subset \mathcal{B}_{c(l d)}$, we have

$$
\theta(l d)=\left[\varepsilon_{1}, \varepsilon_{2}, \ldots, \varepsilon_{n_{x}}\right]
$$

where $\bar{x}_{l d}$ is defined by

$$
\begin{aligned}
& \left\{\begin{array}{l}
\bar{x}_{0}=0, \\
\bar{x}_{k}=\breve{x}_{k}, k \neq l d, \\
\bar{x}_{l d}=E \breve{x}_{l d-1}+D f\left(\breve{x}_{l d-1}\right)+B u_{l d-1},
\end{array}\right. \\
& \left\{\begin{array}{l}
\breve{x}_{0}=0 \\
\breve{x}_{k+1}=E \breve{x}_{k}+D f\left(\breve{x}_{k}\right)+B u_{k}, k \neq l d-1, \\
\breve{x}_{l d}=\bar{x}_{l d}+\hbar_{c(l d)}\left(\varepsilon_{1}, \varepsilon_{2}, \ldots, \varepsilon_{n_{x}}\right) .
\end{array}\right.
\end{aligned}
$$

Decoder:

$$
\left\{\begin{array}{l}
\breve{x}_{0}=0, \\
\breve{x}_{k+1}=E \breve{x}_{k}+D f\left(\breve{x}_{k}\right)+B u_{k}, k \neq l d-1, \\
\breve{x}_{l d}=\bar{x}_{l d}+\hbar_{c(l d)}\left(\varepsilon_{1}, \varepsilon_{2}, \ldots, \varepsilon_{n_{x}}\right) .
\end{array}\right.
$$

Remark 3: It is obvious from (24) that, for an encoding period $[l d,(l+1) d)$, a series of decoded state (denoted by $\breve{X}(l d) \triangleq\{\breve{x}(l d), \breve{x}(l d+1), \ldots, \breve{x}(l+1) d-1\})$ is generated, which means that the decoder can indeed produce the decoded states at both encoding instant $l d$ and those non-encoding time instants $l d+1, \ldots,(l+1) d-1$. Here, the decoded state $\breve{X}(k), k \in(l h,(l+1) d)$ generated at non-encoding instants can be regarded as a "prediction" of the true states of the system.

The following notations are defined for presentation convenience.

$$
\begin{gathered}
c(d) \triangleq \gamma_{2} \epsilon_{0}+\gamma_{1}^{d} \epsilon_{0}, \\
c((l+1) d) \triangleq \gamma_{2}^{l} \epsilon_{0}\left(\gamma_{1}^{d}+\gamma_{2}\right)+\gamma_{1}^{d} \frac{\sqrt{n_{x}} c(l d)}{q} .
\end{gathered}
$$

Lemma 4: The encoding-decoding procedure (22)-(24) satisfies the following constraint:

$$
\left\|\hat{x}_{l d}-\bar{x}_{l d}\right\|_{\infty} \leq c(l d), \quad l=1,2, \ldots
$$

Proof: The proof is carried out by mathematical induction.

1) For $l=1$, by using the property of vector norm, we know that $\left\|\hat{x}_{d}-\bar{x}_{d}\right\|_{2} \leq\left\|\hat{x}_{d}-x_{d}\right\|_{2}+\left\|x_{d}-\bar{x}_{d}\right\|_{2}$, and it then follows from Lemmas 2 and 3 that

$$
\begin{aligned}
& \left\|\hat{x}_{d}-\bar{x}_{d}\right\|_{2} \\
\leq & \left\|\hat{x}_{d}-x_{d}\right\|_{2}+\left\|x_{d}-\bar{x}_{d}\right\|_{2} \\
\leq & \cdots \\
\leq & \gamma_{2} \epsilon_{0}+\gamma_{1}^{d} \epsilon_{0}
\end{aligned}
$$

which guarantees

$$
\left\|\hat{x}_{l d}-\bar{x}_{l d}\right\|_{\infty} \leq c(l d) .
$$

2) Assuming $\left\|\hat{x}_{h d}-\bar{x}_{h d}\right\|_{\infty} \leq c(h d)$ holds for all $h=$ $2,3, \ldots, l$, one immediately has

$$
\begin{aligned}
& \left\|\hat{x}_{(l+1) d}-\bar{x}_{(l+1) d}\right\|_{2} \\
\leq & \left\|\hat{x}_{(l+1) d}-x_{(l+1) d}\right\|_{2}+\left\|x_{(l+1) d}-\bar{x}_{(l+1) d}\right\|_{2} \\
\leq & \cdots \\
\leq & \gamma_{2}^{l+1} \epsilon_{0}+\gamma_{1}^{d}\left\|x_{l d}-\breve{x}_{l d}\right\|_{2} .
\end{aligned}
$$

According to the dynamics of $\breve{x}_{l d}$ in (24), it is further derived that

$$
\begin{aligned}
& \left\|x_{l d}-\breve{x}_{l d}\right\|_{2} \\
= & \left\|x_{l d}-\bar{x}_{l d}-\hbar_{c(l d)}\left(\varepsilon_{1}, \varepsilon_{2}, \ldots, \varepsilon_{n_{x}}\right)\right\|_{2} \\
\leq & \left\|\hat{x}_{l d}-\bar{x}_{l d}-\hbar_{c(l d)}\left(\varepsilon_{1}, \varepsilon_{2}, \ldots, \varepsilon_{n_{x}}\right)\right\|_{2}+\left\|x_{l d}-\hat{x}_{l d}\right\|_{2} \\
\leq & \gamma_{2}^{l} \epsilon_{0}+\frac{\sqrt{n_{x}} c(l d)}{q} .
\end{aligned}
$$

Combining (28) and (29), we draw the conclusion that

$$
\begin{aligned}
& \left\|\hat{x}_{(l+1) d}-\bar{x}_{(l+1) d}\right\|_{2} \\
\leq & \gamma_{2}^{l} \epsilon_{0}\left(\gamma_{1}^{d}+\gamma_{2}\right)+\gamma_{1}^{d} \frac{\sqrt{n_{x}} c(l d)}{q} .
\end{aligned}
$$

Moreover, by noting (28), (30) also implies

$$
\left\|\hat{x}_{(l+1) d}-\bar{x}_{(l+1) d}\right\|_{\infty} \leq c((l+1) d) .
$$


Finally, from (28)-(31), it is straightforward to see that (27) is satisfied for $l \geq 1$, and the proof is now complete.

In Lemma 4, it has been demonstrated that the decoding condition $\hat{x}_{l d}-\bar{x}_{l d} \in \mathcal{B}_{c(l d)}$ holds for all positive integers $l$. Subsequently, a sufficient condition for ensuring the detectability of (1) will be presented in the following theorem which imposes quantitative requirement on the network bandwidth.

Theorem 1: The nonlinear system (1) is detectable under the EDCM (22)-(24) if the following inequality

$$
\frac{\gamma_{1}^{d} \sqrt{n_{x}}}{q}<1
$$

holds where the parameters $\gamma_{1}, d$ and $q$ have been defined in Lemma 2, Lemma 3 and the uniform quantization scheme (7)-(8), respectively.

Proof: Considering the definition of $c(l d)$ and the fact that $0<\gamma_{2}<1$, we see that $\lim _{l \rightarrow \infty} c(l d)=0$. Then, it is inferred from (29) that

$$
\lim _{l \rightarrow \infty}\left\|x_{l d}-\breve{x}_{l d}\right\|_{2}=0
$$

Moreover, noticing that $x_{k}$ and $\breve{x}_{k}$ are actually the trajectories of (1) for those non-encoding instants $k \in(l d,(l+1) d)$, it follows from Lemma 2 that

$$
\left\|x_{k}-\breve{x}_{k}\right\|_{2}^{2} \leq \gamma_{1}^{k-l d}\left\|x_{l d}-\breve{x}_{l d}\right\|_{2}^{2} .
$$

Consequently, we know that $\left\|x_{k}-\breve{x}_{k}\right\|_{2}$ is bounded at the nonencoding instants, which signifies that the nonlinear system (1) is detectable, namely, $\lim _{k \rightarrow \infty}\left\|x_{k}-\breve{x}_{k}\right\|_{2}=0$. The proof is now complete.

In Theorem 1, a sufficient condition is provided for data reconfiguration which means that, if the condition $q>\gamma_{1}^{d} \sqrt{n_{x}}$ is satisfied, then the encoded data can be successfully restored to the true values. Therefore, for a certain $q$, the communication channel is required to be capable of transmitting $\left\lceil\log \left(n_{x} q+1\right)+1\right\rceil$ bits of data at each time instant.

\section{B. Decoder-based controller design}

Lemma 5: [18] Assume that there exist an ISS-Lyapunov function $V\left(k, \rho_{k}\right):[0,+\infty) \times \mathbb{R}^{n} \mapsto \mathbb{R}$, a $\mathcal{K}$ class function $\vartheta(\cdot)$ and three $\mathcal{K}_{\infty}$ class functions $\sigma_{1}(\cdot), \sigma_{2}(\cdot)$ and $\sigma_{3}(\cdot)$ such that the following two inequalities

$$
\sigma_{1}\left(\left\|\rho_{k}\right\|_{2}\right) \leq V\left(k,\left\|\rho_{k}\right\|_{2}\right) \leq \sigma_{2}\left(\left\|\rho_{k}\right\|_{2}\right)
$$

and

$$
V\left(k+1, \rho_{k+1}\right)-V\left(k, \rho_{k}\right) \leq-\sigma_{3}\left(\left\|\rho_{k}\right\|_{2}\right)+\vartheta\left(\left\|\nu_{k}\right\|_{2}\right)
$$

hold for all $\rho_{k} \in \mathbb{R}^{n}$ and $\nu_{k} \in \mathbb{R}^{p}$. Then, the nonlinear discrete-time system (11) is input-to-state stable. Furthermore, the functions $\alpha(\cdot, \cdot)$ and $\beta(\cdot)$ in Definition 3 can be chosen as

$$
\alpha(\cdot, k)=\sigma_{1}^{-1}\left(\psi^{k} \sigma_{2}(\cdot)\right), \quad 0<\psi<1
$$

and

$$
\beta(\cdot)=\sigma_{1}^{-1}\left(\sigma_{2}\left(\sigma_{3}^{-1}(\vartheta(\cdot))\right)\right)
$$

where $\sigma_{1}^{-1}(\cdot)$ stands for the inverse function of the monotone function $\sigma_{1}(\cdot)$ and so does $\sigma_{3}^{-1}(\cdot)$.
According to the decoded state $\breve{x}_{k}$, the decoder-based controller is given as

$$
u_{k}=K_{c} \breve{x}_{k}
$$

where $K_{c}$ is the controller gain to be determined.

Next, let $w_{k} \triangleq \breve{x}_{k}-x_{k}$ be the decoding error vector. In light of (33) and $\breve{x}_{k}=w_{k}+x_{k}$, the closed-loop system (1) is rewritten as

$$
x_{k+1}=\left(E+B K_{c}\right) x_{k}+D f\left(x_{k}\right)+B K_{c} w_{k} .
$$

On the basis of the detectability analysis, we know that the decoding error $w_{k}$ is bounded. In this context, $w_{k}$ can be regarded as a bounded input of (34) and, consequently, the ISS theory can be introduced to develop the encoding-decodingbased control scheme.

Theorem 2: Under the condition in Theorem 1, the decoderbased control system (34) is input-to-state stable if there exist positive definite matrices $Q>0$ and $Z>0$, matrices $G_{11}$, $G_{12}, G_{22}$ and $\bar{K}_{c}$, and a positive scalar $\mu_{3}$ satisfying

$$
\tilde{\Pi}=\left[\begin{array}{cccc}
-Q+\mu_{3} U^{T} U & * & * & * \\
0 & -\mu_{3} I & * & * \\
0 & 0 & -Z & * \\
G \Gamma E+\tilde{K}_{c} & G \Gamma D & \tilde{K}_{c} & \tilde{\Pi}_{0}
\end{array}\right]<0
$$

where

$$
\begin{gathered}
\tilde{\Pi}_{0} \triangleq Q-G \Gamma-\Gamma^{T} G^{T}, \quad \Gamma \triangleq\left[B\left(B^{T} B\right)^{-1}\left(B^{T}\right)^{\perp}\right]^{T}, \\
G \triangleq\left[\begin{array}{cc}
G_{11} & G_{12} \\
0 & G_{22}
\end{array}\right], \quad \tilde{K}_{c} \triangleq\left[\begin{array}{c}
\bar{K}_{c} \\
0
\end{array}\right], \quad \bar{K}_{c} \triangleq G_{11} K_{c} .
\end{gathered}
$$

Furthermore, the controller gain matrix is expressed as

$$
K_{c}=G_{11}^{-1} \bar{K}_{c} .
$$

Proof: Choosing the following ISS-Lyapunov function

$$
V_{k}=x_{k}^{T} Q x_{k}
$$

we obtain

$$
\begin{aligned}
\triangle V_{k}= & V_{k+1}-V_{k} \\
= & {\left[\left(E+B K_{c}\right) x_{k}+D f\left(x_{k}\right)+B K_{c} w_{k}\right]^{T} Q } \\
& \times\left[\left(E+B K_{c}\right) x_{k}+D f\left(x_{k}\right)+B K_{c} w_{k}\right]-x_{k}^{T} Q x_{k} \\
\leq & {\left[\left(E+B K_{c}\right) x_{k}+D f\left(x_{k}\right)+B K_{c} w_{k}\right]^{T} Q } \\
& \times\left[\left(E+B K_{c}\right) x_{k}+D f\left(x_{k}\right)+B K_{c} w_{k}\right] \\
& -x_{k}^{T} Q x_{k}-\mu_{3} f^{T}\left(x_{k}\right) f\left(x_{k}\right)+\mu_{3} x_{k}^{T} U^{T} U x_{k} \\
= & \eta_{3 k}^{T} \Pi \eta_{3 k}+w_{k}^{T} Z w_{k}
\end{aligned}
$$

where

$$
\begin{aligned}
& \eta_{3 k} \triangleq\left[\begin{array}{ccc}
x_{k}^{T} & f^{T}\left(x_{k}\right) & w_{k}^{T}
\end{array}\right]^{T}, \\
& \Pi \triangleq\left[\begin{array}{ccc}
\Pi_{11} & * & * \\
\Pi_{21} & \Pi_{22} & * \\
\Pi_{31} & \Pi_{32} & \Pi_{33}
\end{array}\right], \\
& \Pi_{11} \triangleq\left(E+B K_{c}\right)^{T} Q\left(E+B K_{c}\right)-Q+\mu_{3} U^{T} U, \\
& \Pi_{21} \triangleq D^{T} Q\left(E+B K_{c}\right), \quad \Pi_{22} \triangleq D^{T} Q D-\mu_{3} I, \\
& \Pi_{31} \triangleq\left(B K_{c}\right)^{T} Q\left(E+B K_{c}\right), \quad \Pi_{32} \triangleq\left(B K_{c}\right)^{T} Q D, \\
& \Pi_{33} \triangleq\left(B K_{c}\right)^{T} Q\left(B K_{c}\right)-Z .
\end{aligned}
$$


According to

$$
\begin{aligned}
& G \Gamma+\Gamma^{T} G^{T}-G \Gamma Q^{-1} \Gamma^{T} G^{T}-Q \\
= & -(G \Gamma-Q) Q^{-1}(G \Gamma-Q)^{T} \leq 0,
\end{aligned}
$$

one has

$$
Q-G \Gamma-\Gamma^{T} G^{T} \geq-G \Gamma Q^{-1} \Gamma^{T} G^{T} .
$$

In terms of Schur Complement Lemma and inequalities (35)-(36), we know that $\Pi<0$. Consequently, it is clear that $V_{k+1}-V_{k} \leq-\lambda_{\min }(-\Pi)\left\|x_{k}\right\|_{2}^{2}+\lambda_{\max }(Q)\left\|w_{k}\right\|_{2}^{2}$, and it is then inferred from Lemma 5 that the closed-loop system (34) is input-to-state stable by selecting

$$
\begin{aligned}
\vartheta\left(\left\|w_{k}\right\|_{2}\right) & =\lambda_{\max }(Z)\left\|w_{k}\right\|_{2}^{2}, \\
\sigma_{1}\left(\left\|x_{k}\right\|_{2}\right) & =\lambda_{\min }(Q)\left\|x_{k}\right\|_{2}^{2}, \\
\sigma_{2}\left(\left\|x_{k}\right\|_{2}\right) & =\lambda_{\max }(Q)\left\|x_{k}\right\|_{2}^{2}, \\
\sigma_{3}\left(\left\|x_{k}\right\|_{2}\right) & =\lambda_{\min }(-\Pi)\left\|x_{k}\right\|_{2}^{2} .
\end{aligned}
$$

Letting

$$
\begin{aligned}
\alpha\left(\left\|x_{0}\right\|_{2}, k\right) & =\sqrt{\frac{\psi^{k} \lambda_{\max }(Q)\left\|\epsilon_{0}\right\|_{2}^{2}}{\lambda_{\min }(Q)}}, \\
\beta\left(\left\|w_{k}\right\|_{2}\right) & =\sqrt{\frac{\lambda_{\max }(Q) \lambda_{\max }(Z)\left\|w_{k}\right\|_{2}^{2}}{\gamma \lambda_{\min }(Q) \lambda_{\min }(-\Pi)}}
\end{aligned}
$$

with $0<\gamma<1$, we have from Definition 3 that

$$
\left\|x_{k}\right\|_{2} \leq \alpha\left(\left\|x_{0}\right\|_{2}, k\right)+\beta\left(\left\|w_{k}\right\|_{2}\right),
$$

which completes the proof.

Remark 4: It can be seen from (32) that there is a clear trade-off between the encoding period $d$, the number of the quantization intervals $q$ and the error convergence indicator $\gamma_{1}$. It is often the case that the channel capacity is limited, and therefore adjusting $d$ and $q$ won't be a preferred option. Fortunately, the parameter $\gamma_{1}$ can be made as small as necessary to meet (32) as long as $\gamma_{1}$ lies within the interval $(0,1)$ subject to (14).

Remark 5: In view of the analysis results presented in Theorem 1, the decoding error $\zeta(k)$ can be viewed as the exogenous bounded inputs. As such, in Theorem 2, an effective decoder-based control scheme has been designed such that the ISS of the system (34) can be guaranteed. In addition, in order to solve the controller gain design problem, the orthogonal decomposition is employed. We introduce a free matrix $G$ with a unique structure and construct a matrix $\Gamma=\left[B\left(B^{T} B\right)^{-1} \quad\left(B^{T}\right)^{\perp}\right]^{T}$ so as to cope with the coupling term $Q B K_{c}$ in Theorem 2.

Remark 6: Until now, the outlier-resistant observer-based control problem has been tackled for a class of NSs under the EDCM. The distinctive features with our main results are outlined as follows: 1) the negative effects from the measurement outliers are reduced by constructing a dedicated outlier-resistant observer; 2) the EDCM is employed for data transmission in order to reduce communication resource occupation and enhance the data security; 3 ) the interplay between the network bandwidth, the encoding accuracy and the error convergence is quantitatively analyzed; and 4) the existence condition of the decoder-based controller is parameterized by means of the solution to a certain matrix inequality.

\section{NumericAl EXAMPLE}

In this section, to emphasize the effectiveness of the proposed outlier-resistant observer-based controller design scheme, we consider the following discrete-time nonlinear system (1) with parameters given by:

$$
\begin{aligned}
& E=\left[\begin{array}{ll}
1.05 & -1.0 \\
0.05 & -0.8
\end{array}\right], \quad D=\left[\begin{array}{cc}
0.2 & -0.6 \\
0.2 & 0.4
\end{array}\right], \\
& B=\left[\begin{array}{l}
-1.2 \\
-0.8
\end{array}\right], \quad N=\left[\begin{array}{ll}
1.85 & -0.4
\end{array}\right] .
\end{aligned}
$$

The nonlinear function is selected as

$$
f\left(x_{k}\right)=\left[\begin{array}{c}
0.05 x_{1, k}-\tanh \left(0.05 x_{1, k}\right) \\
0.2 x_{2, k}
\end{array}\right] .
$$

It is readily seen that (2) is satisfied with $U=$ $\operatorname{diag}\{0.1,0.1\}$. The saturation function $\sigma\left(N e_{k}\right)$ is described as follows:

$$
\sigma\left(N e_{k}\right)=\left\{\begin{array}{cl}
N e_{k}, & \text { if }-e_{\max } \leq N e_{k} \leq e_{\max }, \\
e_{\max }, & \text { if } N e_{k} \geq e_{\max } \\
-e_{\max }, & \text { if } \quad N e_{k} \leq-e_{\max }
\end{array}\right.
$$

where the saturation value is taken as $e_{\max }=0.1$.

Example 1: The aim of this example is to verify the detectability of the nonlinear system (1). In this case, it is generally assumed that $u_{k}=0$. Choosing $\varrho_{1}=0.8, \varrho_{2}=0.9$ and $d=3$, we solve the matrix inequalities (15) and (18) to obtain $\gamma_{1}=3.4267$ and $\gamma_{2}=0.3423$. Moreover, the observer gain matrix $K_{e}$ is calculated as $K_{e}=\left[\begin{array}{ll}-1.4002 & 0.0917\end{array}\right]^{T}$. The simulation results are displayed in Figs. 2-5, where Fig. 2 characterizes the abnormal disturbances added to $y_{k}$, Figs. 34 plot the actual states of $x_{1, k}$ and $x_{2, k}$, their estimates $\hat{x}_{1, k}$ and $\hat{x}_{2, k}$, and their decoded values $\breve{x}_{1, k}$ and $\breve{x}_{2, k}$, and Fig. 5 depicts the corresponding decoding errors $w_{1, k}$ and $w_{2, k}$. It is easy to see from Fig. 5 that the errors between the actual states of the network and their decoded states asymptotically approach zero, which indicates that the desired detectability performance of the addressed NSs is well attained.

Example 2: The second example is given to test the validity of the designed decoder-based controller for the nonlinear system (1). The control input signal is taken as the form of (33). By solving (35) in Theorem 2, we obtain the desired controller parameter as $K_{c}=G_{11}^{-1} \bar{K}_{c}=\left[\begin{array}{ll}0.6286 & -0.8525\end{array}\right]$.

As stated in Theorem 2, the considered NS should achieve the ISS with the designed controller parameters given above. Such a theoretical result is confirmed by the simulation results presented in Figs. 6-8.

Example 3: In the third example, to further illustrate the superiority of our proposed outlier-resistant observer, a traditional observer is given as follows for the purpose of comparison:

$$
\left\{\begin{aligned}
\hat{x}_{k+1} & =E \hat{x}_{k}+D f\left(\hat{x}_{k}\right)+B u_{k}+\tilde{K}_{e}\left(y_{k}-H \hat{x}_{k}\right) \\
\hat{x}_{0} & =0
\end{aligned}\right.
$$

where $\tilde{K}_{e}$ is the observer gain matrix.

Based on the traditional observer, the simulation results are exhibited in Figs. 9-11. It is obvious to see that the traditional observer is no longer effective with the appearance of measurement outliers, and this further shows the advantage of our proposed theoretical results. 


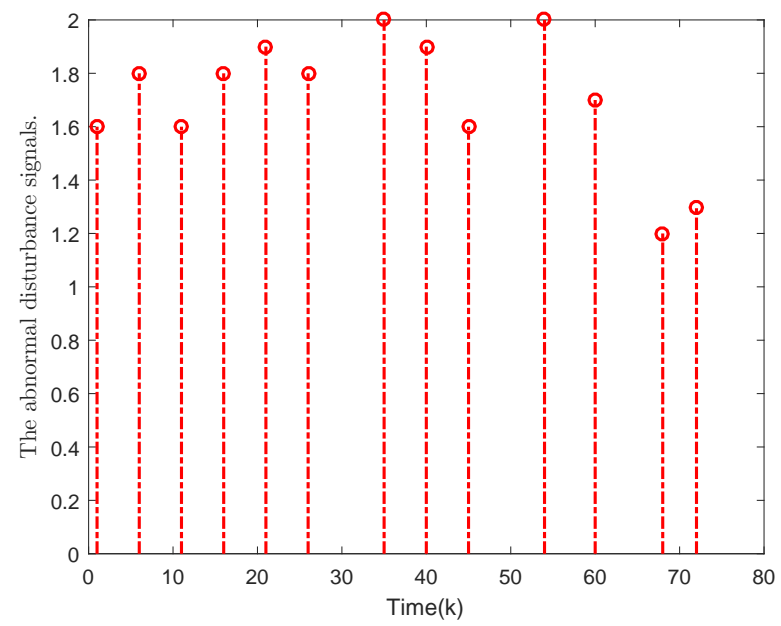

Fig. 2. The abnormal disturbance signals

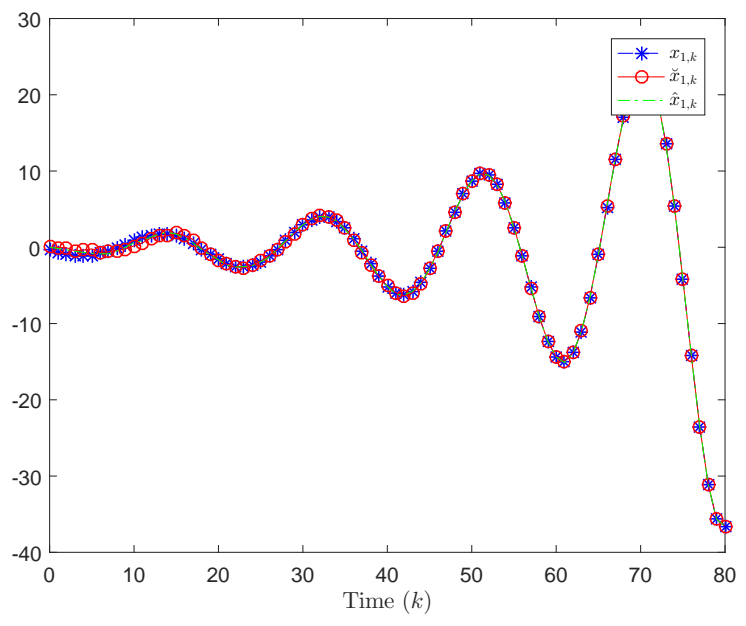

Fig. 3. $x_{1, k}$, its estimate $\hat{x}_{1, k}$ and its decoding value $\breve{x}_{1, k}$ with innovation constraint $\left(u_{k}=0\right)$

\section{CONCLUSiOns}

In this paper, considering the appearance of measurement outliers, the outlier-resistant observer-based control problem has been tackled for a class of NSs under the EDCM. A specific saturation function has been introduced in the state observer to mitigate the negative effects of measurement outliers on the error dynamics of the observation. With the help of the uniform quantization technique, the EDCM has been utilized in the observer-to-controller channel to realize the data compression and therefore reduce the communication resource occupation and enhance the data security. Based on the decoded data, an observer-based control scheme has been put forward. In terms of the solution to a certain matrix inequality constraint, a sufficient condition has been obtained such that the ISS of the closed-loop system can be guaranteed. Finally, three numerical examples have been conducted to verify the effectiveness and superiority of the proposed outlier-resistant observer-based control scheme. As a future research topic, the encoding-decoding-based estimation problem deserves further

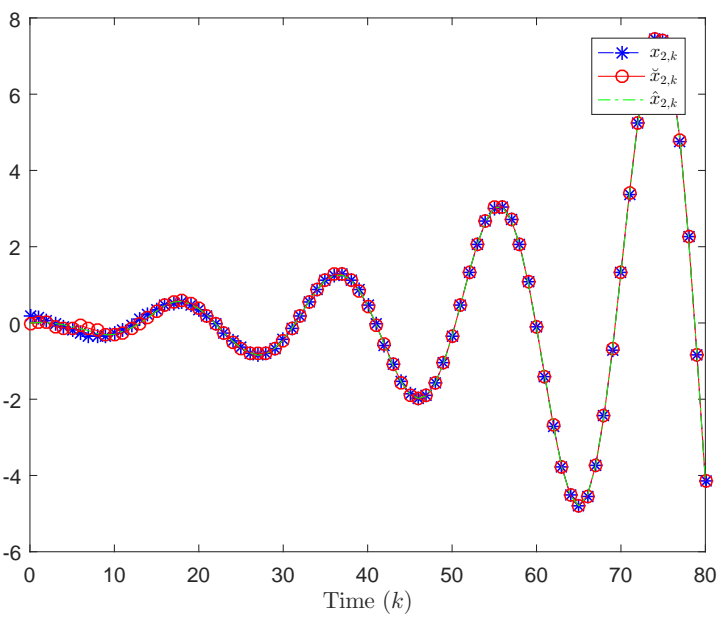

Fig. 4. $x_{2, k}$, its estimate $\hat{x}_{2, k}$ and its decoding value $\breve{x}_{2, k}$ with innovation constraint $\left(u_{k}=0\right)$

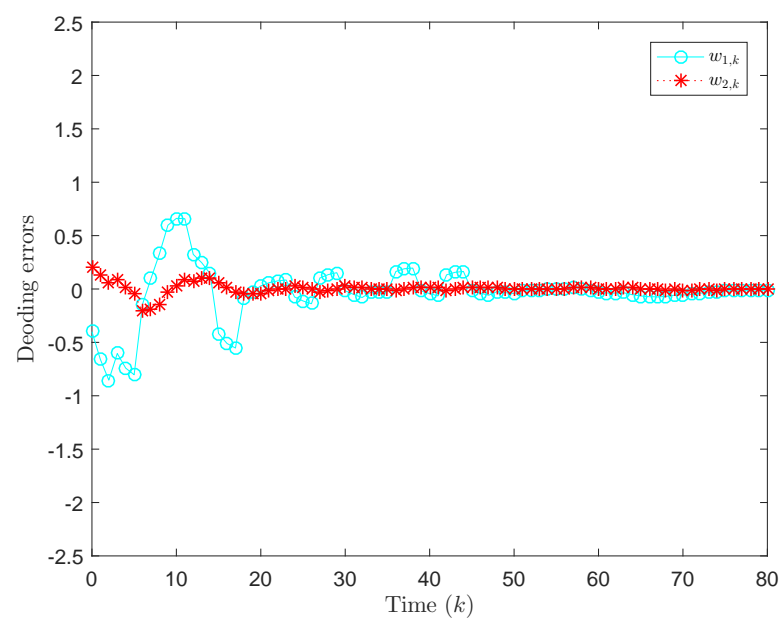

Fig. 5. Decoding errors $w_{1, k}$ and $w_{2, k}$ with innovation constraint $\left(u_{k}=0\right)$

investigation especially for more complicated systems [11], [19], [39].

\section{REFERENCES}

[1] D. A. Akkaya and M. L. Tiku, Robust estimation in multiple linear regression model with non-Gaussian noise, Automatica, vol. 44, pp. 407 417, 2008.

[2] A. Alessandri and M. Awawdeh, Moving-horizon estimation with guaranteed robustness for discrete-time linear systems and measurements subject to outliers, Automatica, vol. 67, pp. 85-93, 2016.

[3] A. Alessandri and L. Zaccarian, Stubborn state observers for linear timeinvariant systems, Automatica, vol. 88, pp. 1-9, 2018.

[4] D. Ciuonzo, A. Aubry, and V. Carotenuto, Rician MIMO channel- and jamming-aware decision fusion, IEEE Transactions on Signal Processing, vol. 65, no. 15, pp. 3866-3880, 2017.

[5] Y. Cui, Y. Liu, W. Zhang and F. E. Alsaadi, Sampled-based consensus for nonlinear multiagent systems with deception attacks: The decoupled method, IEEE Transactions on Systems, Man, and Cybernetics-Systems, in press, DOI: 10.1109/TSMC.2018.2876497.

[6] D. Ding, Z. Wang, D. W. C. Ho and G. Wei, Observer-based eventtriggering consensus control for multiagent systems with lossy sensors and cyber-attacks, IEEE Transactions on Cybernetics, vol. 47, no. 8, pp. 1936-1947, 2016. 


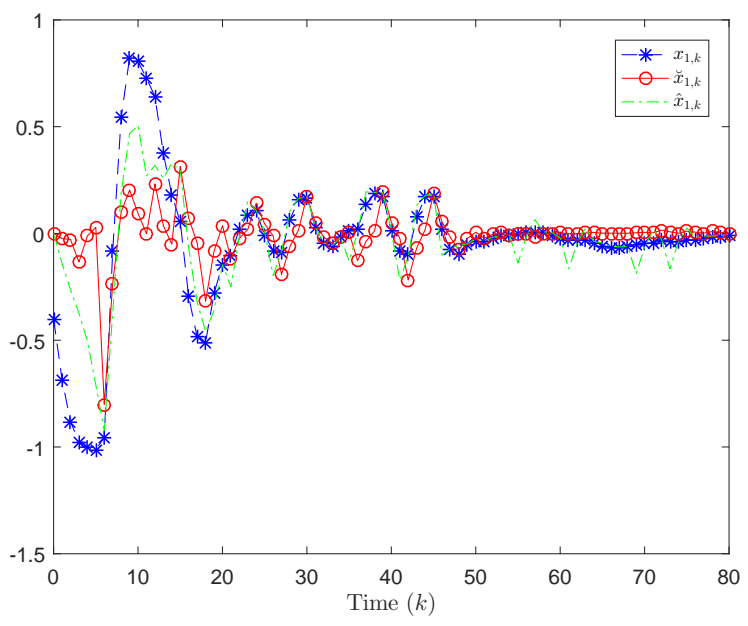

Fig. 6. $x_{1, k}$, its observed value $\hat{x}_{1, k}$ and its decoding value $\breve{x}_{1, k}$ with innovation constraint $\left(u_{k} \neq 0\right)$

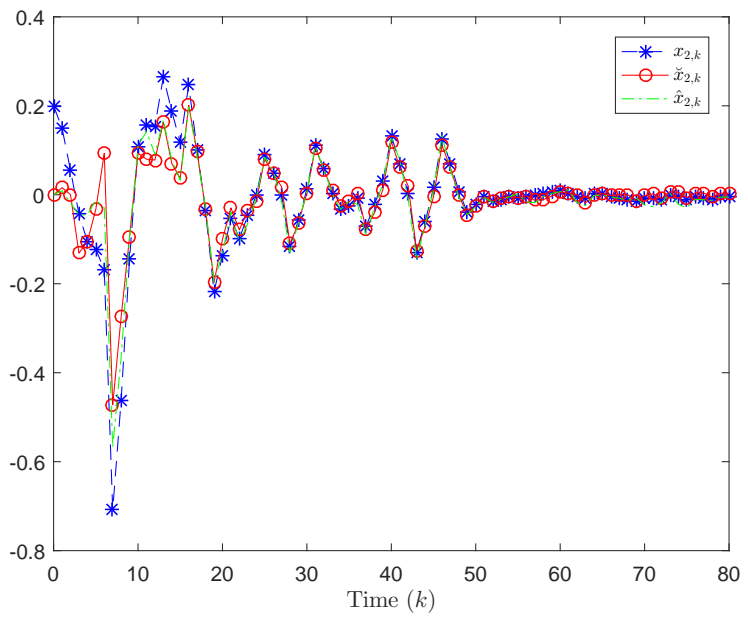

Fig. 7. $x_{2, k}$, its observed value $\hat{x}_{2, k}$ and its decoding value $\breve{x}_{2, k}$ with innovation constraint $\left(u_{k} \neq 0\right)$

[7] D. Ding, Z. Wang and Q.-L. Han, Neural-network-based consensus control for multiagent systems with input constraints: The event-triggered case, IEEE Transactions on Cybernetics, vol. 50, no. 8, pp. 3719-3730, Aug. 2020.

[8] H. Dong, N. Hou and Z. Wang, Fault estimation for complex networks with randomly varying topologies and stochastic inner couplings, Automatica, vol. 112, art. no. 108734, 11 pages, 2020.

[9] H. Dong, Z. Wang and H. Gao, Fault detection for Markovian jump systems with sensor saturations and randomly varying nonlinearities, IEEE Transactions on Circuits and Systems I: Regular Papers, vol. 59, no. 10, pp. 2354-2362, 2012.

[10] M. A. Gandhi and L. Mili, Robust Kalman filter based on a generalized maximum-likelihood-type estimator, IEEE Transactions on Signal Processing, vol. 58, pp. 2509-2520, 2010.

[11] H. Geng, Y. Liang, Y. Liu, and F. E. Alsaadi, Bias estimation for multirate sensor fusion with unknown inputs, Information Fusion, vol. 39, pp. 139-153, 2018.

[12] K. Han, J. Luo, Y. Liu and A. V. Vasilakos, Algorithm design for data communications in duty-cycled wireless sensor networks: A survey, IEEE Communications Magazine, vol. 51. no. 7, pp. 107-113, 2013.

[13] W. P. M. H. Heemels, A. R. Teel, N. Van de Wouw and D. Nešić, Networked control systems with communication constraints: Tradeoffs between transmission intervals, delays and performance, IEEE Transactions on Automatic Control, vol. 55, no. 8, pp. 1781-1796, Aug. 2010.

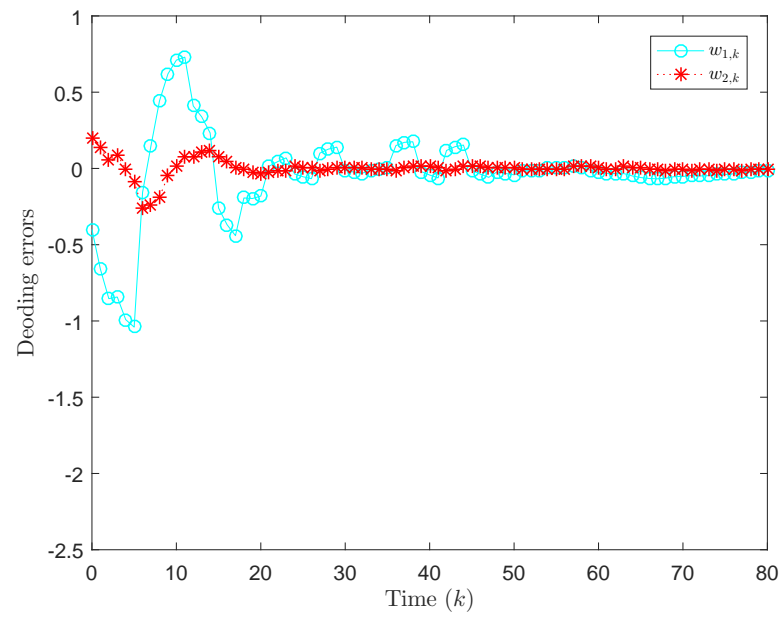

Fig. 8. Decoding errors $w_{1, k}$ and $w_{2, k}$ with innovation constraint $\left(u_{k} \neq 0\right)$

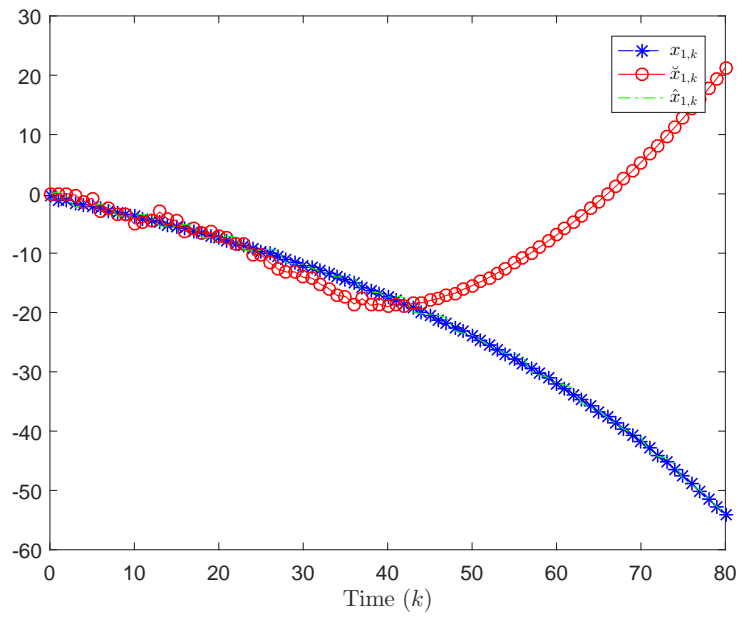

Fig. 9. $x_{1, k}$, its observed value $\hat{x}_{1, k}$ and its decoding value $\breve{x}_{1, k}$ without innovation constraint $\left(u_{k} \neq 0\right)$

[14] N. Hou, Z. Wang, D. W. C. Ho and H. Dong, Robust partial-nodes-based state estimation for complex networks under deception attacks, IEEE Transactions on Cybernetics, vol. 50, no. 6, pp. 2793-2802, Jun. 2020.

[15] J. Hu, Z. Wang, G.-P. Liu, H. Zhang and R. Navaratne, A predictionbased approach to distributed filtering with missing measurements and communication delays through sensor networks, IEEE Transactions on Systems, Man, and Cybernetics-Systems, in press, DOI: 10.1109/TSMC.2020.2966977.

[16] J. Hu, Z. Wang, G.-P. Liu, C. Jia and J. Williams, Event-triggered recursive state estimation for dynamical networks under randomly switching topologies and multiple missing measurements, Automatica, vol. 115, Art. no. 108908, May. 2020.

[17] Y. Jiang and S. Yin, Recent advances in key-performance-indicator oriented prognosis and diagnosis with a MATLAB toolbox: DB-KIT, IEEE Transactions on Industrial Informatics, vol. 15, no 5, pp. 28492858,2018

[18] Z. P. Jiang and Y. Wang, Input-to-state stability for discrete-time nonlinear systems, Automatica, vol. 37, no, 6, pp. 857-869, 2001.

[19] J. Li, H. Dong, Z. Wang and X. Bu, Partial-neurons-based passivityguaranteed state estimation for neural networks with randomly occurring time-delays, IEEE Transactions on Neural Networks and Learning Systems, vol. 31, no. 9, pp. 3747-3753, 2020.

[20] L. Li, M. Chadli, S. X. Ding, J. Qiu and Y. Yang, Diagnostic observer design for t-s fuzzy systems: Application to real-time-weighted faultdetection approach, IEEE Transactions on Fuzzy Systems, vol. 26, no. 2, 


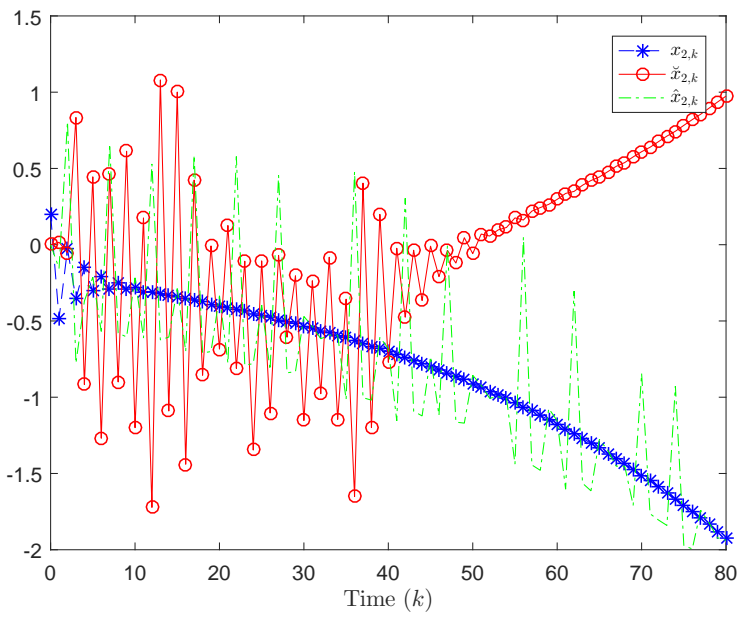

Fig. 10. $x_{2, k}$, its observed value $\hat{x}_{2, k}$ and its decoding value $\breve{x}_{2, k}$ without innovation constraint $\left(u_{k} \neq 0\right)$

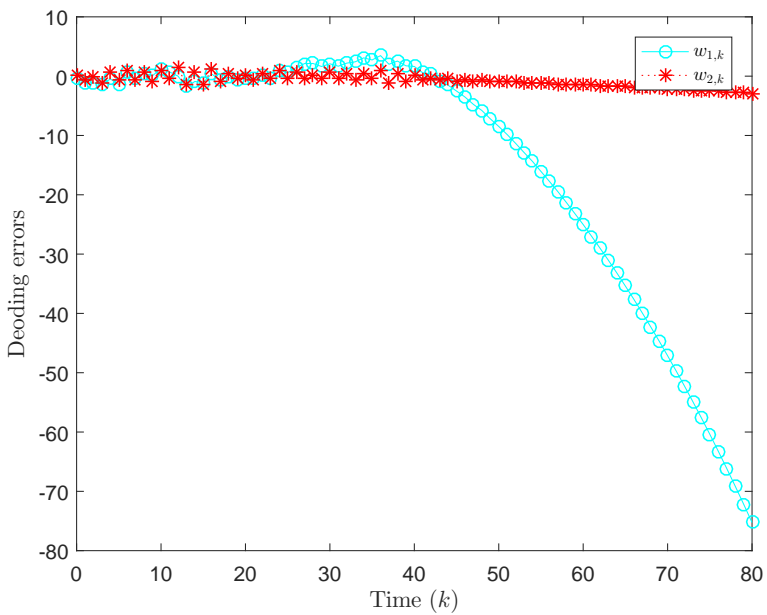

Fig. 11. Decoding errors $w_{1, k}$ and $w_{2, k}$ without innovation constraint $\left(u_{k} \neq\right.$ $0)$

pp. 805-816, 2017.

[21] X. Li, H. Dong, Z. Wang and F. Han, Set-membership filtering for state-saturated systems with mixed time-delays under weighted try-oncediscard protocol, IEEE Transactions on Circuits and Systems II: Express Briefs, vol. 66, no. 2, pp. 312-316, 2018.

[22] J. Liang, H. Chen and J. Lam, An improved criterion for controllability of boolean control networks, IEEE Transactions on Automatic Control, vol. 62, no. 11, pp. 6012-6018, 2017.

[23] D. Liberzon, On stabilization of linear systems with limited information, IEEE Transactions on Automatic Control, vol. 48, no. 2, pp. 304-307, 2003.

[24] D. Liberzon, J. P. Hespanha, Stabilization of nonlinear systems with limited information feedback, IEEE Transactions on Automatic Control, vol. 50, no. 6, pp. 910-915, 2005.

[25] D. Liu, Z. Wang, Y. Liu and F. E. Alsaadi, Recursive resilient filtering for nonlinear stochastic systems with packet disorders, Journal of The Franklin Institute, vol. 357, no. 8, pp. 4817-4833, May 2020.

[26] H. Liu, Z. Wang, B. Shen and H. Dong, Delay-distribution-dependent $H_{\infty}$ state estimation for discrete-time memristive neural networks with mixed time-delays and fading measurements, IEEE Transactions on Cybernetics, vol. 50, no. 2, pp. 440-451, Feb. 2020.

[27] Q. Liu, Z. Wang, Q.-L. Han and C. Jiang, Quadratic estimation for discrete time-varying non-Gaussian systems with multiplicative noises and quantization effects, Automatica, vol. 113, Art. no. 108714, Mar. 2020
[28] S. Liu, Z. Wang, G. Wei and M. Li, Distributed set-membership filtering for multi-rate systems under the Round-Robin scheduling over sensor networks, IEEE Transactions on Cybernetics, vol. 50, no. 5, pp. 19101920, May. 2020.

[29] S. Liu, Z. Wang, Y. Chen and G. Wei, Protocol-based unscented Kalman filtering in the presence of stochastic uncertainties, IEEE Transactions on Automatic Control, vol. 65, no. 3, pp. 1303-1309, Mar. 2020.

[30] Y. Liu, Z. Wang, H. Dong and H. Liu, Anti-disturbance filter design for a class of stochastic systems with fading channels, Science China Information Sciences, vol. 63, no. 11, Art. no. 219205, Nov. 2020.

[31] Y. Liu, Z. Wang, Y. Yuan and W. Liu, Event-triggered partial-nodesbased state estimation for delayed complex networks with bounded distributed delays, IEEE Transactions on Systems, Man, and CyberneticsSystems, vol. 49, no. 6, pp. 1088-1098, Jun. 2019.

[32] E. Mousavinejad, F. Yang, Q.-L. Han and L. Vlacic, A novel cyber attack detection method in networked control systems, IEEE Transactions on Cybernetics, vol. 48, no. 11, pp. 3254-3264, 2018.

[33] H. Park, Outlier-resistant high-dimensional regression modelling based on distribution-free outlier detection and tuning parameter selection, Journal of Statistical Computation and Simulation, vol. 87, no. 9, pp. 1799-1812, 2017.

[34] B. Shen, Z. Wang, D. Wang and Q. Li, State-saturated recursive filter design for stochastic time-varying nonlinear complex networks under deception attacks, IEEE Transactions on Neural Networks and Learning Systems, vol. 31, no. 10, pp. 3788-3800, Oct. 2020.

[35] B. Shen, Z. Wang, D. Wang and H. Liu, Distributed state-saturated recursive filtering over sensor networks under Round-Robin protocol, IEEE Transactions on Cybernetics, vol. 50, no. 8, pp. 3605-3615, Aug. 2020.

[36] Y. Shen, Z. Wang, B. Shen and F. E. Alsaadi, Nonfragile $H_{\infty}$ filtering for discrete multirate time-delayed systems over sensor networks characterized by Gilbert-Elliott models, International Journal of Robust and Nonlinear Control, vol. 30, no. 8, pp. 3194-3214, May. 2020.

[37] J. Song, Y. Niu, J. Lam and Z. Shu, A hybrid design approach for output feedback exponential stabilization of Markovian jump systems, IEEE Transactions on Automatic Control, vol. 63, no. 5, pp. 1404-1417, 2018.

[38] D. F. Vecchia and J. D. Splett, Outlier-resistant methods for estimation and model fitting, ISA Transactions, vol. 33, no. 4, pp. 411-420, 1994.

[39] X. Wan, Z. Wang, Q.-L. Han and M. Wu, A recursive approach to quantized $H_{\infty}$ state estimation for genetic regulatory networks under stochastic communication protocols, IEEE Transactions on Neural Networks and Learning Systems, vol. 30, no. 9, pp. 2840-2852, Sept. 2019.

[40] D. Wang, Z. Wang, B. Shen and Q. Li, $H_{\infty}$ finite-horizon filtering for complex networks with state saturations: The weighted try-once-discard protocol, International Journal of Robust and Nonlinear Control, vol. 29, no. 7, pp. 2096-2111, 2019.

[41] L. Wang, Z. Wang, Q.-L.-Han and G. Wei, Synchronization control for a class of discrete-time dynamical networks with packet dropouts: A coding-decoding-based approach, IEEE Transactions on Cybernetics, vol. 48, no. 8, pp. 2437-2448, 2017.

[42] L. Wang, Z. Wang, G. Wei and F. E. Alsaadi, Observer-based consensus control for discrete-time multiagent systems with coding-decoding communication protocol, IEEE Transactions on Cybernetics, vol. 99, pp. 1-11, 2018.

[43] M. Wang, Z. Wang, Y. Chen and W. Sheng, Event-based adaptive neural tracking control for discrete-time stochastic nonlinear systems: A triggering threshold compensation strategy, IEEE Transactions on Neural Networks and Learning Systems, vol. 31, no. 6, pp. 1968-1981, Jun. 2020.

[44] G. Wei, S. Liu, Y. Song and Y. Liu, Probability-guaranteed setmembership filtering for systems with incomplete measurements, $\mathrm{Au}$ tomatica, vol. 60, pp. 12-16, 2015.

[45] Y. Yuan, Z. Wang, P. Zhang and H. Liu, Near-optimal resilient control strategy design for state-saturated networked systems under stochastic communication protocol, IEEE Transactions on Cybernetics, vol. 49, no. 8, pp. 3155-3167, 2018.

[46] Z. Zhang and J. Dong, Observer-based interval type-2 $L_{2}-L_{\infty} / H_{\infty}$ mixed fuzzy control for uncertain nonlinear systems under measurement outliers, IEEE Transactions on Systems, Man, and Cybernetics: Systems, doi: 10.1109/TSMC.2020.2980361, under press.

[47] L. Zou, Z. Wang, H. Geng and X. Liu, Set-membership filtering subject to impulsive measurement outliers: a recursive algorithm, IEEE/CAA Journal of Automatica Sinica, vol. 8, no. 2, pp. 1-12, Feb. 2021.

[48] L. Zou, Z. Wang, Q.-L. Han and D. Zhou, Moving horizon estimation of networked nonlinear systems with random access protocol, IEEE Transactions on Systems, Man, and Cybernetics - Systems, in press, DOI: $10.1109 /$ TSMC.2019.2918002. 
[49] L. Zou, Z. Wang, Q.-L. Han and D. Zhou, Moving horizon estimation for networked time-delay systems under Round-Robin protocol. IEEE Transactions on Automatic Control, vol. 64, no. 12, pp. 5191-5198, 2019.

[50] L. Zou, Z. Wang and D. Zhou, Moving horizon estimation with non-uniform sampling under component-based dynamic event-triggered transmission, Automatica, vol. 120, art. no. 109154, 13 pages, Oct. 2020.

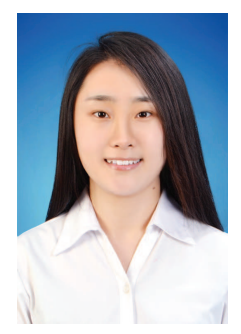

Jiahui Li received the B.E. degree in automation from Northeast Petroleum University, Daqing, China, in 2015. She is currently pursuing the Ph.D. degree in petroleum and natural gas engineering at the Northeast Petroleum University, Daqing, China.

From 2018 to 2019, she was a Visiting Scholar with the Department of Computer Science, Brunel University London, London, U.K. Her current research interests include networked control systems, and oil gas information and control engineering.

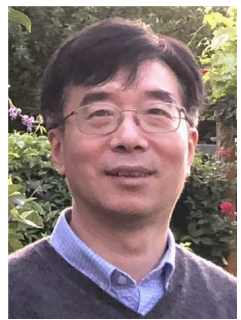

Zidong Wang (SM'03-F'14) was born in Jiangsu, China, in 1966. He received the B.Sc. degree in mathematics in 1986 from Suzhou University, Suzhou, China, and the M.Sc. degree in applied mathematics in 1990 and the Ph.D. degree in electrical engineering in 1994, both from Nanjing University of Science and Technology, Nanjing, China.

$\mathrm{He}$ is currently Professor of Dynamical Systems and Computing in the Department of Computer Science, Brunel University London, U.K. From 1990 to 2002 , he held teaching and research appointments in universities in China, Germany and the UK. Prof. Wang's research interests include dynamical systems, signal processing, bioinformatics, control theory and applications. He has published 250+ papers in IEEE Transactions and $60+$ papers in Automatica. He is a holder of the Alexander von Humboldt Research Fellowship of Germany, the JSPS Research Fellowship of Japan, William Mong Visiting Research Fellowship of Hong Kong.

Prof. Wang serves (or has served) as the Editor-in-Chief for Neurocomputing, the Deputy Editor-in-Chief for International Journal of Systems Science, and an Associate Editor for 12 international journals including IEEE Transactions on Automatic Control, IEEE Transactions on Control Systems Technology, IEEE Transactions on Neural Networks, IEEE Transactions on Signal Processing, and IEEE Transactions on Systems, Man, and CyberneticsPart C. He is a Member of the Academia Europaea, a Fellow of the IEEE, a Fellow of the Royal Statistical Society and a member of program committee for many international conferences.

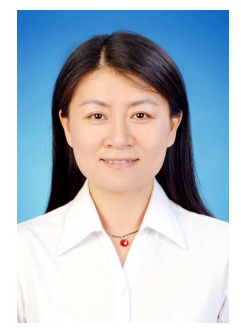

Hongli Dong received the Ph.D. degree in control science and engineering from the Harbin Institute of Technology, Harbin, China, in 2012.

From 2009 to 2010, she was a Research Assistant with the Department of Applied Mathematics, City University of Hong Kong, Hong Kong. From 2010 to 2011, she was a Research Assistant with the Department of Mechanical Engineering, The University of Hong Kong, Hong Kong. From 2011 to 2012, she was a Visiting Scholar with the Department of Information Systems and Computing, Brunel University London, London, U.K. From 2012 to 2014, she was an Alexander von Humboldt Research Fellow with the University of Duisburg-Essen, Duisburg, Germany. She is currently a Professor with the Artificial Intelligence Energy Research Institute, Northeast Petroleum University, Daqing, China. She is also the Director of the Heilongjiang Provincial Key Laboratory of Networking and Intelligent Control, Daqing. Her current research interests include robust control and networked control systems.

Dr. Dong is a very active reviewer for many international journals.

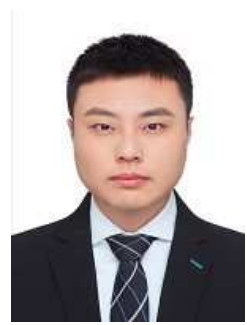

Xiaojian Yi was born in 1987. He received the B.S. degree in control technology from the North University of China in 2010, and M.S. degree in reliability engineering from the Beijing Institute of Technology in 2012 and the Ph.D. degree in reliability engineering from Beijing Institute of Technology in 2016. And during the 2015-2016, he is as Joint training $\mathrm{PhD}$ in University of Ottawa to study on robot reliability and maintenance. From 2016 to 2020, he was an Associate Professor with the China North Vehicle Research Institute, and until now, he is an Associate Professor with the Beijing Institute of Technology. He is the author of two books, more than 100 articles, and holds 8 patents. His research interests include system reliability analysis and intelligent control, fault diagnosis and health management. 